\title{
Finite Element based Model of Modified Niagara Foot and its Effect on Stiffness
}

\author{
Ahmed H. Kandil \\ Systems and Biomedical Engineering Department \\ Faculty of Engineering-Cairo University
}

Giza, Egypt

\begin{abstract}
A prosthetic foot-ankle system is designed with the purpose of improving the amputee gait by storing and releasing elastic energy during the stance stage of the gait. Such foot performance is based on its structural stiffness characteristics. Choosing the appropriate stiffness of a prosthetic foot is planned to substitute the loss of the muscles and tendons of the intact foot. The aims of this study are to study the effect of the thickness of the upper part of the S-shape and removing the prongs on the structural stiffness of a modified Niagara foot using the FEA. In this work, a simulated model based on finite element is built. Then, an analysis is conducted on the modified models of Niagara foot using the boundary conditions of the ISO-10328 and expanded using the boundary conditions of the ISO-22675 to study the effect of removing the prongs from the models. The stiffness results of the FEA are compared by considering the $\mathrm{K} 1, \mathrm{~K} 2$, and $\mathrm{KH}$ values. The prongs of the Niagara foot had a major contribution in force-displacement relationships. So, by removing these prongs, the stresses imposed in a foot due to loading the toe exceeded the yielding stress for the feet when proposing the Delrin 2700 or Hytrel 8238 materials. Also the absence of the prongs showed remarkable effects on the toe stiffness variation with the simulated time of stance, especially at the earlier stages of the toe contact where this effect diminished gradually up to the toe-off.
\end{abstract}

\section{General Terms}

Biomechanics.

\section{Keywords}

Finite Element; Niagara foot; Stiffness.

\section{INTRODUCTION}

An important aspect of a prosthetic foot that strongly influences its function is the structural stiffness (resistance to deformation) [1]. Naturally, the biological limb can change the stiffness of the joints through the extent of muscle activation, while the prosthetic limb does not have this ability. Therefore, the design of the prosthetic limb must take into account its stiffness characteristics in order to substitute for the loss of the tendons and muscles of the intact biological limb [2]. The structural stiffness as a characteristic of the prosthetic foot is not a single value, since it is not only based on the design, and the material of foot, but also on the attitude of loading which changes during the walk [3,4].

The Prosthetic foot evaluation demands study of both functional and mechanical behaviors [5]. The functional behavior of prosthetic feet has commonly been evaluated using gait analysis. The majority of the gait studies of amputees concentrated on comparing the effect of type of prosthetic foot on the kinematics and kinetics parameters [68]. The mechanical behavior of prosthetic feet has commonly been evaluated by measuring the hysteresis and stiffness of different prosthetic feet at several ankle positions that shows an extensive variety of mechanical properties [5], Also, because of the high costs and difficulties of experimental studies, Finite Element analysis (FEA) can be used to evaluate new and/or modified designs of the prosthetic feet. This technique has the advantage of testing new designs, even before manufacturing a prototype of the foot. The FEA can permit the investigation of the reactions of the deformable structures using known boundary conditions [5]. It has been generally applied to measure the stresses between the residual limb and the socket [9-11]. Few studies have concentrated on the analysis of the prosthetic foot at the stance phase of the gait cycle $[12,13]$. In fact, using the FEA needs data about geometry, boundary conditions of the structure, and the material properties [5, 14].

There are two main standards used in performing the mechanical testing of prosthetic feet, ISO-10328 and ISO22675 prostheses $[15,16]$ which include the protocols to experimentally test the durability and performance of the lower-limb prostheses. The ISO-10328 prescribed four sequential testing sections prostheses [17]: an initial static proof test, an ultimate strength test, a cyclic test and a final static proof test. While ISO-22675, outlines a cyclic durability testing procedures for the lower limb prosthetic devices. It provides M-shape curves, which are the plots of both, the testing forces and the tilting angles of the loading platform upon a prosthetic foot versus time. This plot provides practical testing regimes [12] designed to simulate conditions during the stance phase of prosthetic gait.

The prescription of appropriate design characteristics [18] (e.g., stiffness) is not well-defined. Several studies, [8, 19-21] have been conducted to evaluate the differences between the commercially available prosthetic feet, to help in understanding and prescribing their functionality. Such feet normally have many mechanical differences, which makes linking functional difference to a specific prosthetic foot feature difficult.

Van Jaasrsveld et al. [1] conducted a study on 9 different prosthetic feet without shoes and with leather, sports shoes. They did not follow the ISO-10328, where the feet were loaded from $-30^{\circ}$ (heel strike position) to $35^{\circ}$ (toe-off position) with increments of one degree. Using a horizontal plate to simulate the ground in all 66 positions then lowering it down on the foot with $1 \mathrm{~mm}$ increases until the deflection reach $35 \mathrm{~mm}$ or the $1000 \mathrm{~N}$ of a vertical force. Results showed that applying leather shoe leads to increase in the maximum stiffness during loading.

Geil [22], tested eleven types of prosthetic feet to provide an independent measurement of material and structural properties including the stiffness. All feet were plantar flexed at $12^{\circ}$, load displacement testing on the foot was vertical compression with a constant displacement rate of $1 \mathrm{~mm} / \mathrm{sec}$ to a maximum force of $800 \mathrm{~N}$. The stiffness of the foot was 
expressed as the slope of the load-deformation curve, where such relationship, was considered to be linear. The feet were classified into one of four categories: the most stiff category (average, $0.0760 \mathrm{~N} / \mathrm{m}$ ), the more stiff category (average, $0.0606 \mathrm{~N} / \mathrm{m}$ ), the less stiff category (average, $0.0384 \mathrm{~N} / \mathrm{m}$ ) and the least stiff category (average, $0.0277 \mathrm{~N} / \mathrm{m}$ ). However, the test did not follow the ISO-10328, where the test was applied only on the toe region, without considering multiple pylon angles and the forces applied did not reflect the peak loading during gait.

Although many of researchers [3-5, 17, 23, 24] have followed the ISO-10328 in performing mechanical tests on different types of prosthetic feet. They evaluated stiffness by different ways. EL-Mohandes, M. [3], studied the effect of thickness variation of the upper part of the S-region, for the Niagara prosthetic foot, on the foot stiffness behavior. Three foot models were fabricated with the same material and dimensions except the thickness "b". The feet were subjected to compressive loading at two different modes of contact, the heel and the forefoot modes. The loading was applied in a set of fixed angles up to $1000 \mathrm{~N}$. These angles were between $-30^{\circ}$ to $0^{\circ}$ and $0^{\circ}$ to $30^{\circ}$, in $10^{\circ}$ increments, for the heel and the forefoot modes respectively. In order to compare stiffness of the feet, the slope of secant lines of the force-displacement curves in the interval of loading between $400 \mathrm{~N}$ and $1000 \mathrm{~N}$ were only considered. This range was considered to cover the major loading range.

Mason, Z. D. et al. [17], conducted a study of performance and robustness, on three different types of feet, according to the ISO-10328. Each of the two foot sections (forefoot and heel) was subjected to a compressive load with a rate of 175 $\mathrm{N} / \mathrm{s}$ and up to $2240 \mathrm{~N}$. The loading was at $20^{\circ}$ and $15^{\circ}$ angles (measured from the vertical axis) for the two foot sections respectively. The results of the initial and the final static proof tests were used to ascertain stiffness and creep experienced by each section. They determined the stiffness as the slope of the linear best-fit of the force-displacement curve during the loading portion of the initial and final static proof test.

Bonnet, X. et al. [5], performed mechanical compressive tests on a J-shape foot. The tests were performed according to ISO10328. The foot was plantar flexed at $20^{\circ}$ simulating push-off and dorsflexed at $15^{\circ}$ simulating heel loading. The load was applied vertically to the foot at a constant rate of $250 \mathrm{~N} / \mathrm{s}$ up to $1300 \mathrm{~N}$. They reproduced the mechanical testing specifications of the ISO-10328 to perform finite element (FE) modeling with vertical displacements until reaching of $1200 \mathrm{~N}$ load. The stiffness specified by both the experimental and the FE model curves were assigned as the linear stiffness. The resulting load-displacement curves showed linear parts in the range of displacement from 0 to $45 \mathrm{~mm}$ in the forefoot loading case and from 0 to $10 \mathrm{~mm}$ in the heel loading case. After this linear part, a non-linearity appeared which resulted from the contact modifications between the foot and the loading plate. The linear stiffness values were calculated for both experimental and finite element model curves. The study exhibited that the FE model curves of the forefoot loading matched with that obtained by the mechanical testing. The corresponding results were less applicable in the heel loading case.

Schmitz, A. [23], conducted a study on the Niagara foot model (1). The study involved finite element (FE) modeling for validating the mechanical test results. Tests were performed according to the ISO-10328, where the foot was subjected to compressive loading up to $1600 \mathrm{~N}$. The loads were generated, by applying displacements to a platen at a rate of $5 \mathrm{~mm} /$ minute, upon the heel and the toe sections through angles of $15^{\circ}$ and $20^{\circ}$ respectively. In order to describe stiffness of the foot, three stiffness parameters K1, $\mathrm{K} 2$ and $\mathrm{KH}$ were considered. The first stiffness parameter (K1) was the slope of the line from the start point of contact (zero load) up to an elbow point in the force-displacement curve. While, $\mathrm{K} 2$ was the slope of a line from the elbow point to $1000 \mathrm{~N}$, and $\mathrm{KH}$ was the slope of line from zero to $1000 \mathrm{~N}$ loads.

Haberman, A. et al. [24], conducted mechanical testing using load conditions typical to that of gait and based on the ones outlined in ISO-10328. The tests were conducted on two models of Niagara foot and on SACH and ESAR commercial feet. The heel and toe portions were tested at contact angles in $5^{\circ}$ increments from $0^{\circ}$ to $20^{\circ}$ and $0^{\circ}$ to $30^{\circ}$ for the heel and toe respectively. Each foot was subjected to compressive loading up to a value of 1.2 times the design load (user body weight recommended by the manufacturer). The force-deflection responses were described in terms of two regions. In the first, the slope of the force-deflection curve was defined as the initial stiffness (S1) of the foot, while two other values (S2, and $\mathrm{Sh}$ ) were used to describe the nonlinear behavior for the rest of the curve. The value $S 2$, determined the slope of the curve of the design load, and the value (Sh) as the average stiffness between $0 \mathrm{~N}$ and the design load. For the toe portion the same protocol was followed and the analogous parameters S3, S4 and St were used. Results showed that the prosthetic feet behaved like non-linear components, whose heel and toe stiffness increased as the applied load increased. Results elucidated that, the stiffness of the feet were dependent on the contact angle of the applied load. They decided that, multiple stiffness values were needed to accurately describe the nonlinear behavior of feet.

Haberman [12], Conducted experimental methodology based on the ISO standardized waveform for evaluating the mechanical properties of the toe and heel parts of prosthetic feet. The purpose of his study was to use the tilt angles of the ISO 22675 testing protocol corresponding with the force displacement testing be conducted on the toe and heel pylon angles. From P4 loading curve 15 point were extracted to simulate the loading conditions. The 15 points (corresponding time and force values) were extracted with $5^{\circ}$ increments for the toe from $0^{\circ}$ to $35^{\circ}$ and for the heel from $-20^{\circ}$ to $0^{\circ}$. Three designs of the feet were tested (SACH foot, Niagara Foot Model 2 Version 18 made of Hytrel and two energy return feet Axtion). All feet were loaded with a peak force equal to the product of their design mass with a displacement rate of $2.0 \mathrm{~mm} / \mathrm{sec}$. The results showed that the displacement profile and stiffness characteristics of the SACH, Niagara Foot and Axtion were compatible with their design features, indicating that the mechanical testing protocol is capable to detect differences in function and able to capture the mechanical characteristics of these designs.

Figueroa and Muller-Karger [13], performed a numerical study on a novel design of a Dynamic Energy Return prosthetic foot applying the methodology suggested by Haberman [12] using FEA and compared the displacement profile and stiffness characteristics of his novel foot with those of Niagara foot, AXTION foot and SACH foot commercial prostheses [13]

Niagara foot is a durable and low cost prosthetic foot. It intends to provide improved performance for the lower limb amputees. The structural stiffness behavior of the Niagara foot is based on its shape, type of material, and the attitude of loading. By changing either the foot material, foot shape or 
both, the stiffness will be altered. The aims of this study are to study the effect of the thickness of the upper part of the Sshape and removing the prongs on the structural stiffness of a modified Niagara foot using the FEA.

This paper is organized as follows. Section two describes the methodology. In section three, the results are presented. Section four includes the discussion. In section five, the conclusions are presented.

\section{METHODOLOGY}

In this study, the numerical stiffness evaluation, of the modified models of Niagara foot, was conducted. These models were created by changing the thickness " $\mathrm{B}$ " of the upper part of S-shape and with removing the prongs. The stiffness values of the heel and toe were evaluated separately considering the boundary conditions of ISO-10328. The test was expanded using the boundary conditions of the ISO22675 to study the effect of removing the prongs from the models.

\subsection{A. Foot shape model}

The Niagara foot was drawn first with the original dimensions using SolidWorks software [23]. Four modified models were created by changing the upper part of S-shape "B" without changing the remaining dimensions, Fig. 1. The thickness "B" was altered by shifting the center of the outer semicircle horizontally from the center 0 by the distances of $5 \mathrm{~mm}, 10$ $\mathrm{mm}$ and $15 \mathrm{~mm}$ to obtain the Models (nominated M1, M2, $\mathrm{M} 3$, and M4 respectively), so the values of "B" were 12.5 , $17.5 \mathrm{~mm}, 22.5 \mathrm{~mm}$ and $27.5 \mathrm{~mm}$ respectively [4]. Other modifications were made on the four models (M1, M2, M3 and M4) which included removing the prongs, In order to study the effects of removing the prongs on the heel and the toe stiffness. The new modified models without prongs were nominated as (MM1, MM2, MM3 and MM4), Fig. 2.

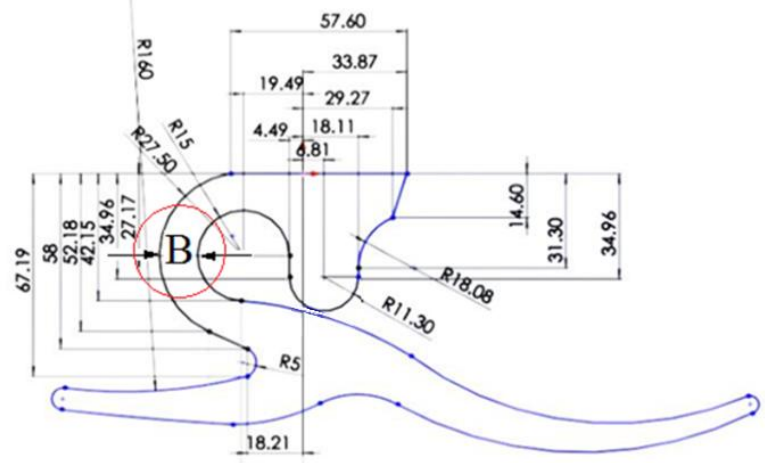

Fig. 1 The thickness "B" of the upper part of the S-shape.

\subsection{Types of material}

Stiffness not only depend on the shape of the model but also on the type of material. Two different materials were used Delrin 2700, and Hytrel 8238 to model the modified Niagara foot on the FEA. Hytrel 8238 was used in different studies $[12,23]$. Delrin 2700 is a new grade of high flow Acetal copolymer with wide manufacturing processes. These materials were assumed to have linear, isotropic, and elastic stress-strain behavior. A summary of the mechanical properties of such materials is shown in Table 1.

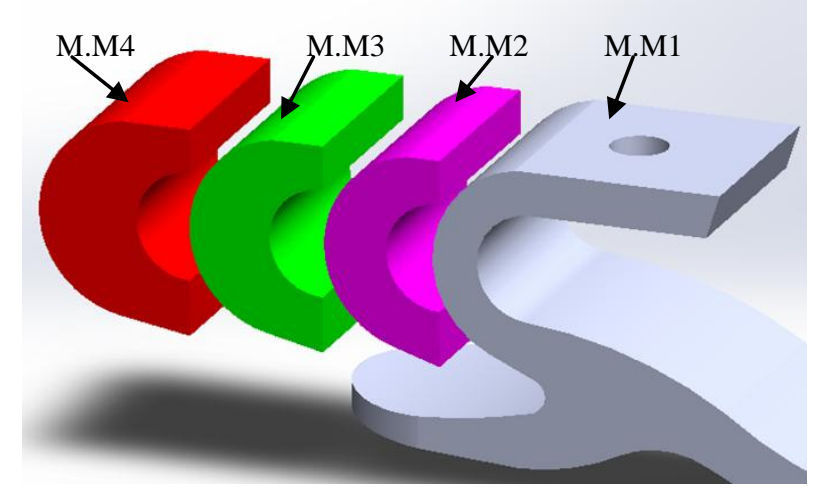

Fig. 2 The four models without prongs (MM1, MM2, MM3, and MM4).

Table 1 Mechanical properties of the used materials [25]

\begin{tabular}{|c|c|c|c|c|c|}
\hline \multirow{2}{*}{$\begin{array}{c}\text { Material } \\
\text { Name }\end{array}$} & \multicolumn{5}{|c|}{ Mechanical Properties } \\
\cline { 2 - 6 } & $\begin{array}{c}\text { Mass } \\
\text { Density } \\
\left(\mathbf{k g}_{\mathbf{m}} \mathbf{3}\right)\end{array}$ & $\begin{array}{c}\text { Poisson's } \\
\text { Ratio }\end{array}$ & $\begin{array}{c}\text { Elastic } \\
\text { Modulus } \\
(\text { MPa })\end{array}$ & $\begin{array}{c}\text { Tensile } \\
\text { Strength } \\
(\mathbf{M P a})\end{array}$ & $\begin{array}{c}\text { Yield } \\
\text { Strength } \\
(\mathbf{M P a})\end{array}$ \\
\hline Delrin2700 & 1410 & 0.3 & 2900 & 40.7 & 63 \\
\hline Hytrel8238 & 1280 & 0.45 & 1180 & 48.3 & 36 \\
\hline
\end{tabular}

\subsection{Boundary conditions}

The boundary conditions of ISO-10328 were used. The loads applied on the heel and on toe portions were created by a circular loading platen against the two foot portions separately. This platen was inclined at $-15^{\circ}$ and $20^{\circ}$ angles with respect to the Tibia axis, to apply loading on the heel and the toe portions respectively. The loading platen was considered as a rigid body. A coordinating system was created on the platen to control its movement, where remote displacements of the platen were generated to provide loads on the heel and toe modes of loading. The coordinate system was originated $(\mathrm{x}=0, \mathrm{y}=0$ and $\mathrm{z}=0)$ in the center of the surface of platen. The platen was allowed to move along the " $y$ " direction only without any rotation. Contact settings between the platen and either the heel or the toe surfaces were treated as frictionless without penetration. Also, the prosthetic foot set to be self-interacted and another contact set was created at the prongs and the opposite face and adopted as frictionless with no penetration. The platform of the foot was set to be fixed (immovable in the $\mathrm{x}, \mathrm{y}$ and $\mathrm{z}$ directions).

Meshing is another step in the design analysis. The SolidWorks program automatically assigns the appropriate mesh type to the object based on its geometry features. It creates a solid mesh with tetrahedral solid elements in the foot solid shape. For all proposed models of the Niagara foot, the SolidWorks assigned different sizes of elements ranged between $6.5 \mathrm{~mm}$ and $7.5 \mathrm{~mm}$. These values then refined to be $5 \mathrm{~mm}$ for all the models of the foot.

The FEA was extended to study effect of removing the prongs on the displacement and stiffness behavior of the foot models during the stance phase. Models 3 with thickness $B=22.5 \mathrm{~mm}$ using the material Delrin 2700 with and without the prongs. The two models were subjected to loading conditions as prescribed by the ISO-22675. Such conditions required to apply certain values of force which were corresponding to the vertical ground reaction loads expected during the stance phase $(600 \mathrm{msec}$.). These values of force, as well as the inclination angles, were proposed from P4 loading curve and that proposed by Haberman [12]. 


\section{RESULTS}

In this part, the FE testing of the modified models, of Niagara foot, were performed based on two different regimes. The first was based on the ISO-10328 standard to study of the effect of the thickness of the upper part of S-shape "B" on models without the prongs and effect of removing the prongs on the model. The stiffness results of the FEA are compared by considering the $\mathrm{K} 1, \mathrm{~K} 2$, and $\mathrm{KH}$ values. The second was based on the ISO-22675 standard to study the effect of removing the prongs on the foot models during the stance phase.

\subsection{The First Numerical Testing Regime (Based on ISO-10328)}

\subsubsection{Effect of the thickness on models without} prong on the heel and toe stiffness

Regarding to the new models: MM1, MM2, MM3, and MM4 (having no prongs), where they were subjected to the same settings and procedures of testing. From the FE testing results, all the new feet models of the Hytrel 8238, imposed stresses exceeding to the material yielding point. Similar observations also were obtained for the two models, MM1 and MM2 when proposing the Delrin 2700. So, these new models were omitted from the study that the results of them would be inconsistent with the hypothesis proposed for the material (linear elastic). Fig. 3, shows the stress distribution (color coded), of model MM1 when proposing the Delrin 2700, where the Von Mises stress reached a maximum value of 96.895 $\mathrm{MPa}$ (yielding point $=63 \mathrm{MPa}$ ). So the analysis was performed only on the two models MM3 and MM4 when proposing the Delrin 2700 as the material of the feet.

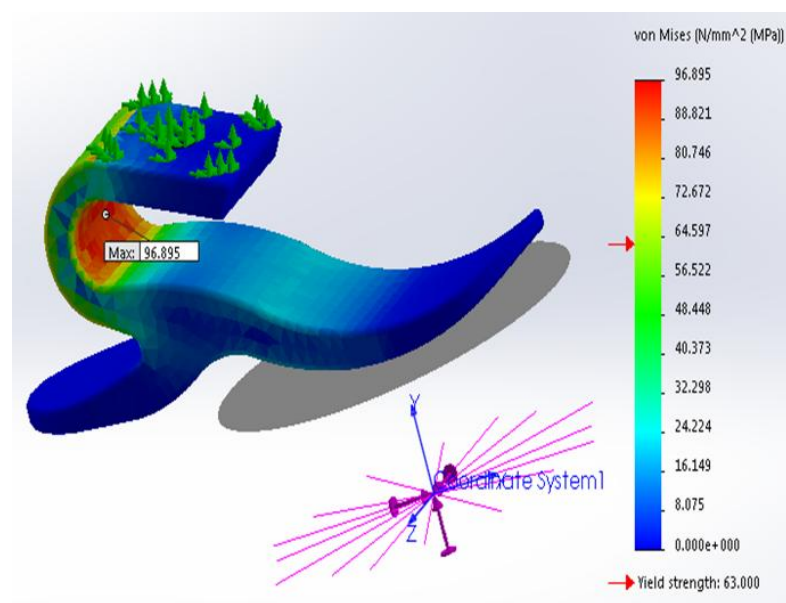

Fig. 3 Von Mises stress distribution for MM1 foot during toe mode of loading (Delrin 2700).

Fig. 4, illustrates a plot of the force-displacement values resulted from the FE tests obtained of modified models (MM3 and MM4) on the heel mode of loading using Delrin 2700. The FE results indicated that the thickness " $\mathrm{B}$ " has a slight effect on the force-displacement behavior of the heel mode of loading, where the values of the stiffness K2 were 199.6 $\mathrm{KN} / \mathrm{m}$ and $203.7 \mathrm{KN} / \mathrm{m}$ for the models two MM3 and MM4 respectively. Such observation could be pointed out to the corresponding values of stiffness $\mathrm{KH}$, while $\mathrm{K} 1$ for the two models were the same. Table 2, shows the stiffness values K1, $\mathrm{K} 2, \mathrm{KH}$ of the heel.

Fig. 5, illustrates a plot of the force-displacement data from the FE testing of the toe of the two modified models MM3 and MM4. This data is relevant to the proposed material of the Delrin 2700. This figure illustrated a clear effect of the thickness "B" on the stiffness behavior of the feet new models MM3, and MM4 (with no prongs), where the MM4 showed higher force/displacement trends. This effect did not arise when testing the corresponding feet M4 and M3 that having prongs. Table 2, shows the stiffness values $\mathrm{K} 1, \mathrm{~K} 2, \mathrm{KH}$ of the toe.

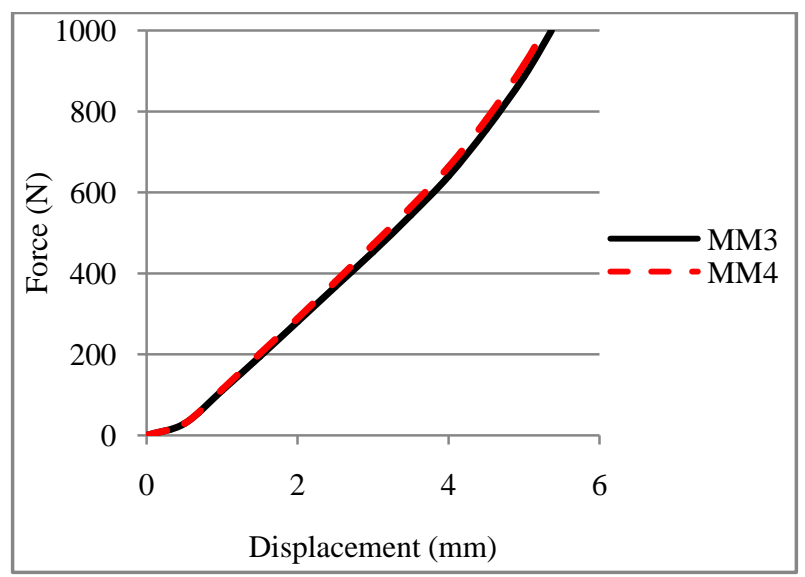

Fig. 4 Effect of thickness "B" on the force-displacement relationship of the foot models MM3 and MM4 at the heel mode of loading (proposing the Delrin 2700 as the material)

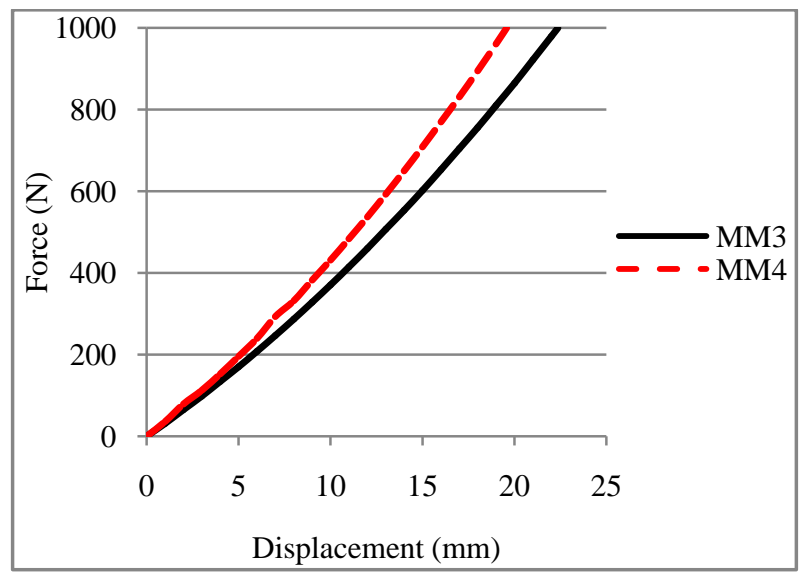

Fig. 5 Effect of thickness "B" on the force-displacement relationship of the foot models MM3 and MM4 at the toe mode of loading (proposing the Delrin 2700 as the material)

Table 2 Stiffness K1, K2, and KH of the foot models MM3 and MM4 at the heel and toe mode of loading (proposing the Delrin 2700 as the material)

\begin{tabular}{|c|c|c|c|c|}
\hline \multirow{2}{*}{ Model } & \multicolumn{3}{c|}{ K values of the heel } \\
\cline { 3 - 5 } \multicolumn{2}{|c|}{} & $\begin{array}{c}\text { K1 } \\
(\mathbf{K N} / \mathbf{m})\end{array}$ & $\begin{array}{c}\text { K2 } \\
(\mathbf{K N} / \mathbf{m})\end{array}$ & $\begin{array}{c}\text { KH } \\
(\mathbf{K N} / \mathbf{m})\end{array}$ \\
\hline \multirow{2}{*}{ Heel } & MM3 & 58.0 & 199.6 & 186.4 \\
\cline { 2 - 5 } & MM4 & 58.4 & 203.7 & 189.9 \\
\hline \multirow{2}{*}{ Toe } & MM3 & 33.6 & 47.1 & 44.7 \\
\cline { 2 - 5 } & MM4 & 38.2 & 38.2 & 51.1 \\
\hline
\end{tabular}

\subsubsection{Effect of the prong's removal on the heel and toe stiffness}

The FE results indicated that removing the prongs from the M3 and M4 show no effect on the force displacement behavior during the heel mode of loading. For MM3 Fig. 6, shows a plot of the force-displacement values of the M3 (having the prongs) and MM3 (having no prongs) in the heel 
mode of loading when proposing Derlin2700. This figure showed that the two models had an identical behavior. Such observation was reflected on the values of stiffness, (Table 3). The existence or absence of the prongs didn't play any role upon the stiffness of the foot in the heel mode of loading.

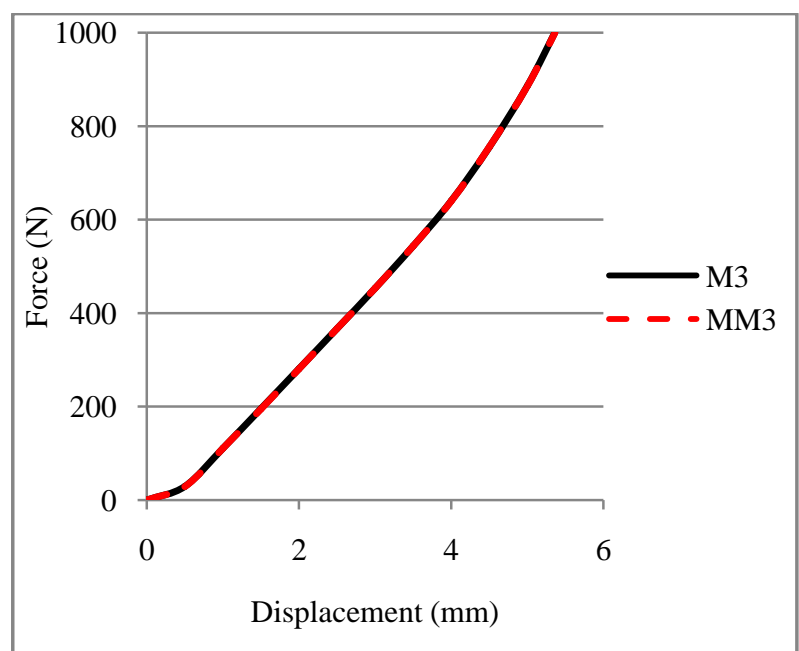

Fig. 6 Effect of removing the prong on the force displacement relationship of the foot models M3 and MM3 at the heel mode of loading.

Fig. 7, illustrates a plot of the force-displacement values resulted from the FE testing obtained for the 3rd models of the foot: with prongs (M3) and without prongs (MM3) where the values of the thickness " $\mathrm{B}$ " were equal for both $(\mathrm{B}=22.5$ $\mathrm{mm}$ ). The material selected for this test was the Delrin 2700 . The maximum displacement of the toe was, $12.25 \mathrm{~mm}$ for the M3, while it was $22.36 \mathrm{~mm}$ for MM3. This clearly illustrated the effect of the existence of prongs on the toe stiffness. The two cures of M3 and MM3 were coincided up to about $3 \mathrm{~mm}$ of the displacement, then the M3 elbow observed beyond this displacement. Table 3, shows the stiffness values K1, K2, and $\mathrm{KH}$ for the two models M3 and MM3. K1 for both models were almost equal, so they did show significant difference. The stiffness $\mathrm{K} 2$, and $\mathrm{KH}$ were more realistic in appointing such effect.

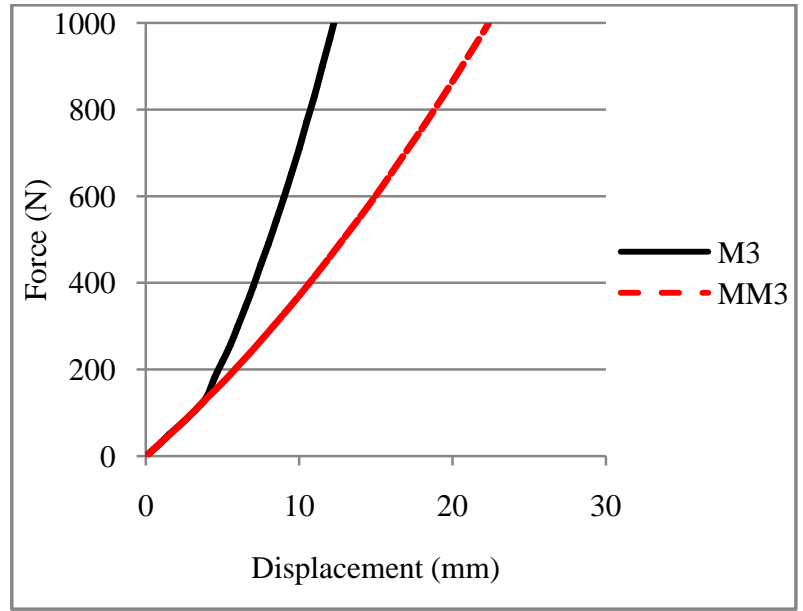

Fig. 7 Effect of removing the prong on the force displacement relationship of the foot models M3 and MM3 at the toe mode of loading.
Table 3 Stiffness K1, K2, and KH of the foot models M3 and MM3 at the heel and toe mode of loading (proposing the Delrin 2700 as the material)

\begin{tabular}{|c|c|c|c|c|}
\hline \multirow{2}{*}{\multicolumn{2}{|c|}{ Model }} & \multicolumn{3}{|c|}{ K values of the heel } \\
\hline & & \multirow{2}{*}{$\begin{array}{c}\mathbf{K} 1(\mathbf{K N} / \mathbf{m}) \\
58.4\end{array}$} & \multirow{2}{*}{$\begin{array}{c}\mathbf{K 2}(\mathbf{K N} / \mathbf{m}) \\
199.8\end{array}$} & \multirow{2}{*}{$\begin{array}{c}\mathbf{K H}(\mathbf{K N} / \mathbf{m}) \\
186.6\end{array}$} \\
\hline Ho & M3 & & & \\
\hline Heel & MM3 & 58.0 & 199.6 & 186.4 \\
\hline \multirow{2}{*}{ Toe } & M3 & 34.6 & 104.4 & 81.6 \\
\hline & MM3 & 33.6 & 47.1 & 44.7 \\
\hline
\end{tabular}

\subsection{The Second Numerical Testing Regime (Based on ISO-22675)}

Fig. 8, shows the predicted displacement-time relationships during the stance period for both, the heel and the toe parts. The plot was performed to the models M3 and MM3, in order to study the effect of removing the prongs of the stiffness behavior of the foot. It is clear from this figure that the prongs had no sensible effect on the heel, while the absence of the prongs showed a remarkable effect on the toe especially at the earlier stages of the toe contact that this effect diminishes gradually up to the toe off. Similar conclusions could be extracted from the study of the two models M4 and MM4.

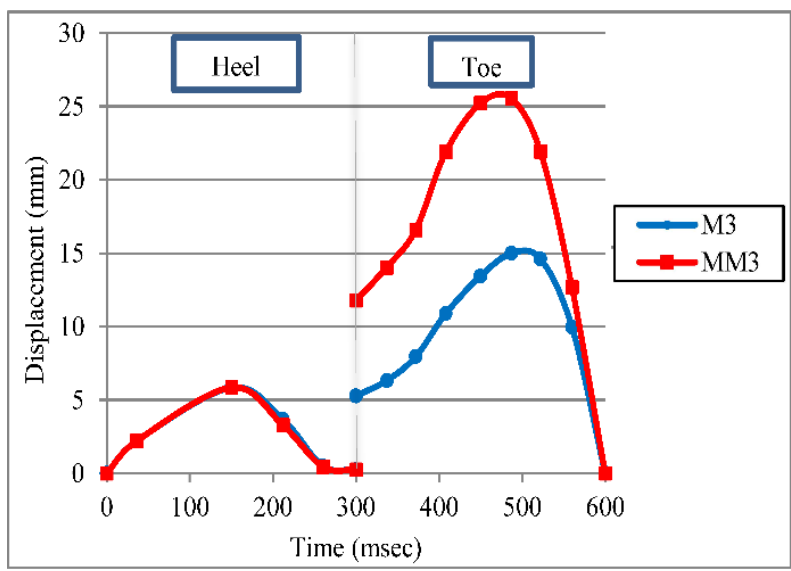

Fig. 8 Prediction of Model 3 displacement with and without prongs during stance phase.

Fig. 9 and Fig. 10, shows the stiffness-time trends of the heel and the toe for the models M3 and MM3. The two models showed similar trends accompanied with some deviations for both the heel and the toe modes of loading. These deviations unnoticeable at the start of stance (heel contact) and at the end of stance (toe off). Similar conclusions could be underlined for the other two models M4 and MM4. 


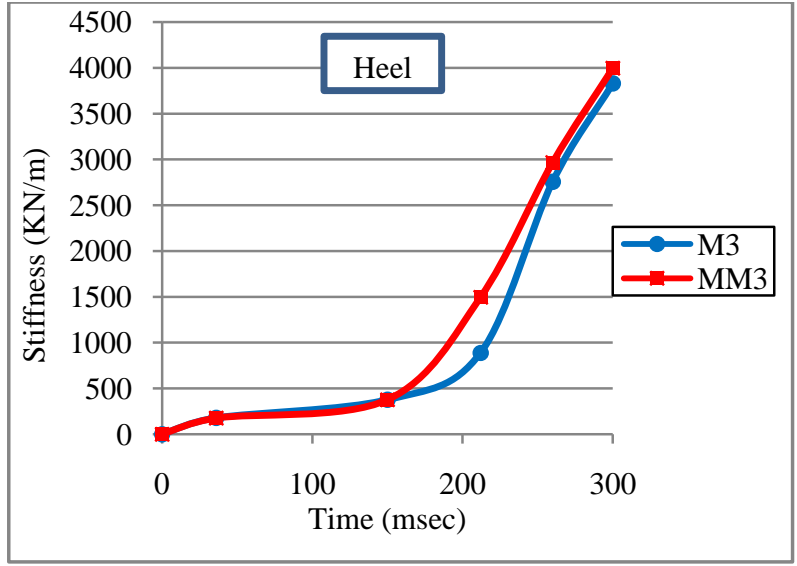

Fig. 9 Prediction of Model 3 stiffness with and without prongs on the heel during stance phase.

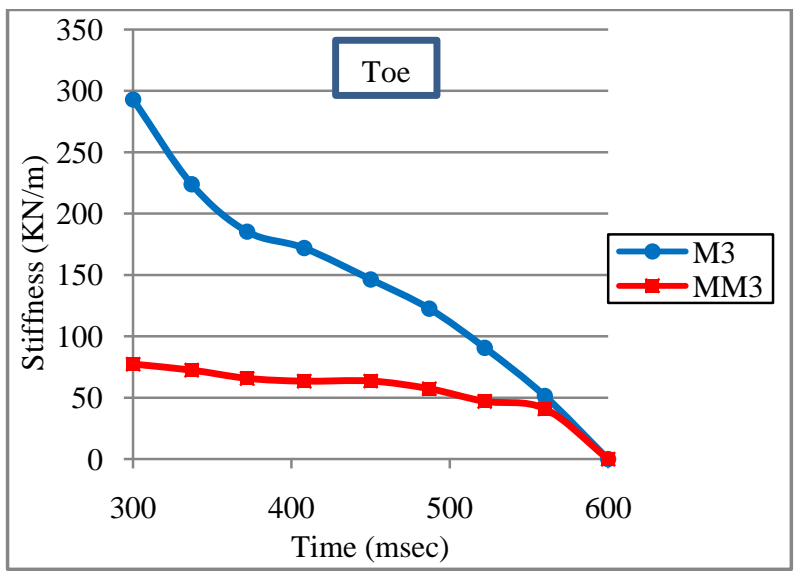

Fig. 10 Prediction of Model 3 stiffness with and without prongs on the toe during stance phase.

\section{DISCUSSION}

For the models without prongs, removing the prongs lead to influence the feet to stresses higher than the material yield stress using the two materials Delrin 2700 and Hytrel 8238. While with increasing the thickness "B" for models MM3 and MM4 using the material Delrin 2700 did not reach the material yield stress. So, the type of material and increasing the thickness played an important role in decreasing the stresses. During the heel loading of the foot, the thickness did not show higher differences in the stiffness values, while increasing the stiffness of the foot during the loading of the toe.

Removing the prongs from model 3 showed that the loaddisplacement relationship of the M3 and MM3 on the heel mode testing were identical. The gap between the arch and the prongs didn't play any role in the load-displacement relationship. At loading the toe, the force-displacement relation showed a rapid change (elbow in the curve) for M3 due to the effect of the bending moment which was in a direction that assisted closing the gap between the prongs and the arch. While with removing the prongs from the model (MM3) this elbow disappeared and the stiffness of the toe decreased. This observation was denoted for the $\mathrm{K} 2$, and $\mathrm{KH}$ stiffness parameters.

For the evaluation of displacements and stiffness at the 15 critical points, according to the ISO-22675, which simulate the loading during the stance phase. Removing the prongs from M3 showed almost identical values for the displacement and stiffness during the heel loading. But at the toe loading mode MM3 showed higher displacement behavior and lower stiffness behavior.

The proposed method in this study covered a wider range of load-displacement behavior, mimicking what could happen in a real prosthetic foot. As it was subjected to different levels of load based on the different conditions of the users' characteristics and the walking process. Moreover, these values resulted from the methodology based on the mathematical model. This methodology can be used to compare the stiffness of prosthetic feet with different designs and different materials.

\section{CONCLUSIONS}

The results of the FE analysis tests showed that, the thickness of the upper part of the S-shape in the Niagara foot "B" had a proportional effect on the toe stiffness when the foot loaded at $20^{\circ}$. The stiffness values $\mathrm{K} 1$ of the four models showed high proportionality with the thickness "B", while such proportionality declined through the progression of loading (K2 and $\mathrm{KH})$. After the early stage of the toe loading (at $20^{\circ}$ angle), the force-displacement behavior exhibited rapid changes (elbow in the curve), which were reflected on the higher values of $\mathrm{K} 2$ and $\mathrm{KH}$.

The prongs of the Niagara foot had a major contribution in the force-displacement relationships. So, by removing these prongs, the stresses imposed in a foot due to loading the toe exceeded the yielding stress for the feet when proposing the Delrin 2700 or Hytrel 8238 materials. This observation was detected for the foot models of the lower " $B$ " values MM1, and MM2, while the other two models of higher "B" values (MM3 and MM4) showed lower stiffness behavior as compared with the feet having prongs (M3 and M4). The absence of the prongs showed remarkable effects on the toe stiffness variation with the simulated time of stance, especially at the earlier stages of the toe contact where this effect diminished gradually up to the toe-off.

\section{REFERENCES}

[1] H. W. L. VanJaarsveld, H. J. Grootenboer, J. DE. Vries, and H. F. J. M. Koopman, "Stiffness and hysteresis properties of some prosthetic feet," Prosthetics and Orthotics International, Vol. 14, No. 3, pp. 117-124, 1990.

[2] G. K. Klute, C. F. Kallfelz, and J. M. Czerniecki, "Mechanical properties of prosthetic limbs: Adapting to the patient," Journal of Rehabilitation Research and Development, Vol. 38, No. 3, pp. 299-307, May/June 2001.

[3] M. El-Mohandes, "Effect of the S-Shape Thickness Variation On The Stiffness Of The Niagara Foot," AlAzhar University Engineering Journal, JAUES, Vol. 9, No. 2, pp. 1-8, Dec. 2014

[4] M. El-Mohandes and M. El.Hussein, "Stiffness Analyses of Modified Niagara Prosthetic Feet Using Finite Element Modelling," in Biomedical Engineering Conference (CIBEC), Cairo, 2014.

[5] X. Bonnet, H. Pillet, P. Fode, F. Lavaste, and W. Skalli, "Finite element modelling of an energy-storing prosthetic foot during the stance phase of transtibial amputee gait," Proceedings of the Institution of Mechanical Engineers, Part H: Journal of Engineering in Medicine, Vol. 226, No. 1, pp. 70-75, Dec. 2011. 
[6] Macfarlane, P.A., Nielsen, D.H., Shurr, D.G. and Meier, K., 1991, "Perception of Walking Difficulty by BelowKnee Amputees Using a Conventional Foot Versus the Flex-Foot", Journal of Prosthetics and Orthotics, Vol.3, No. 3, pp. 114-119.

[7] Macfarlane, P.A., Nielsen, D.H., Shurr, D.G. and Meier, K., 1991, "Gait Comparisons for Below-Knee Amputees Using a Flex-Foot(TM) Versus a Conventional Prosthetic Foot", Journal of Prosthetics and Orthotics, Vol.3, No. 4, pp. 150-161.

[8] Thomas, S.S., Buckon, C.E., Helper, D., Turner, N., Moor, M. and Krajbich, J.I., 2000, "Comparison of the Seattle Lite Foot and Genesis II Prosthetic Foot during walking and running", Journal of Prosthetics and Orthotics, Vol.12, No. 1, pp. 9-14.

[9] Faustini, M.C., Neptune, R.R. and Crawford, R.H., 2006, "The quasi-static response of compliant prosthetic sockets for transtibial amputees using finite element methods", Medical Engineering \& Physics, Vol.28, No. 2 , pp. $114-121$.

[10] Zhang, M., Lord, M., Turner-Smith, A.R. and Roberts, V.C., 1995, "Development of a non-linear finite element modelling of the below-knee prosthetic socket interface", Medical Engineering \& Physics, Vol.17, No. 8, pp. 559566.

[11] Jia, X., Zhang, M., Li, X. and Lee, W.CC. (2005).A quasi-dynamic nonlinear finite element model to investigate prosthetic interface stresses during walking for trans-tibial amputees. Clinical Biomechanics. ; Vol 20, No. 6, pp. 630-635.

[12] Haberman, A., "Mechanical Properties of Dynamic Energy Return Prosthetic Feet”, MSc. Thesis: Queen's University Kingston, Ontario, Canada, 2008

[13] Figueroa, R. and Müller-Karger, C.M., "Using FE for Dynamic Energy Return Analysis of Prosthetic Feet during Desing Process", In 25th Southern Biomedical Engineering Conference, Miami, Florida, USA, 2009, pp. 289-292.

[14] M. Viceconti, S. Olsen, L.-P. Nolte and K. Burton, "Extracting clinically relevant data from finite element simulations," Clinical Biomechanics, Vol. 20, pp. 451454, 2005.
[15] International Organization for Standardization. ISO 10328:2006 Prosthetics: Structural testing of lower-limb prostheses: requirements and test methods.

[16] International Organization for Standardization. ISO 22675:2006 Prosthetics: Testing of ankle-foot devices and foot units: requirements and test methods.

[17] Z. D. Mason, J. Pearlman, R. A. Cooper, and J. Z. Laferrier, "Comparison of prosthetic feet prescribed to active individuals using ISO standards," Prosthetics and Orthotics International, Vol. 35, No. 4, pp. 418-424, Oct. 2011.

[18] H. Goujon, X. Bonnet, P. Sautreuil, M. Maurisset, L. Darmon, P. Fode, and F. Lavaste, "A functional evaluation of prosthetic foot kinematics during lowerlimb amputee gait," Prosthetics and Orthotics International, Vol. 30, No. 2, pp. 213-223, August. 2006.

[19] A. ARYA, "A biomechanical comparison of the SACH, Seattle and Jaipur feet using ground reaction forces," Prosthetics and Orthotics International, Vol. 19, No. 1, pp. 37-45, April 1995.

[20] K. Postema, "Energy storage and release of prosthetic feet Part 1: Biomechanical analysis related to user benefits," Prosthetics and Orthotics International, Vol 21, No. 1, pp. 17-27, April 1997.

[21] K. Postema, "Energy storage and release of prosthetic feet Part 2: Subjective ratings of 2 energy storing and 2 conventional feet, user choice of foot and deciding factor," Prosthetics and Orthotics Internat, Vol. 21, No. 1, pp. 28-34, April 1997.

[22] M. D. Geil, "Energy Loss and Stiffness Properties of Dynamic Elastic Response Prosthetic Feet," Journal of Prosthetics and Orthotics, Vol. 13, No. 3, pp. 70-73, 2001.

[23] A. Schmitz, "Stiffness analyses for the design development of aprosthetic foot", MSc. Thesis: University Of Wisconsin-Madison, 2007.

[24] A. Haberman, T. Bryant, M. Beshai, and R. Gabourie, "Mechanical Characterization Of Prosthetic Feet," in 12th World Congress of the International Society for Prosthetics and Orthotics, Vancouver, Canada, 2007, p. 374.

[25] DuPont Inc. [Online], Available from: http://dupont.materialdatacenter.com/profiler/main/stand ard/main/ds, Nov 2015 